\title{
Bildung für alle
}

\section{Die Umsetzung der Bildungspläne erfordert die Unterstützung der Erzieherinnen}

\author{
Lore Miedaner und Heidi Reinl
}

Prof. Dr. Lore Miedaner ist

Hochschullehrerin für Bildung und

Erziehung in der Kindheit an der

Hochschule Esslingen.

E-Mail Lore.Miedaner@hs esslingen.de

Heidi Reinl ist Forschungsreferentin am

Institut für angewandte Forschung

Gesundheit und Soziales an der

Hochschule Esslingen.

E-Mail Heidi.Reinl@hs esslingen.de
Was Kinder in Kindertagesstätten lernen sollen, ist den staatlichen Bildungsplänen der Bundesländer zu entnehmen. Wie solche Vorgaben in der Praxis umgesetzt werden, zeigt eine Befragung von Erzieherinnen in Baden-Württemberg.

Alle Bundesländer haben mittlerweile Bildungs- oder Orientierungspläne, entsprechende Programme oder Empfehlungen für die pädagogische Arbeit in Kindertageseinrichtungen mit dem Ziel vorgelegt, eine gleichmäßig hohe Qualität in allen Einrichtungen zu erreichen. Für die alten Bundesländer ist dies neu, für die neuen Bundesländer knüpfen solche Vorgaben an den staatlich verordneten Bildungsund Erziehungsplan für Kindergärten in der ehemaligen DDR an, wenngleich heute unter veränderter Ausgangssituation.

Da sowohl die Strukturen von Kindertageseinrichtungen als auch die Inhalte der Bildungspläne (Sammelbegriff für unterschiedliche Bezeichnungen), ihre Reichweite (1) und der Stand ihrer Einführung in den Bundesländern sehr unterschiedlich sind, sind übergreifende Aussagen nur ansatzweise möglich. Bei einer Befragung von Erzieherinnen (2) in BadenWürttemberg wurden jedoch Aspekte deutlich, die vermutlich auch für die Situation von Erzieherinnen in anderen Regionen und Bundesländern gelten können. Einige dieser Aspekte von exemplarischer Bedeutung stellen wir hier dar.

Befragt wurden Erzieherinnen aus sechs Kindergärten in einem Mittelzentrum im Einzugsbereich einer Großstadt. Obwohl es in keiner der Einrichtungen von der Arbeit mit den Kindern freigestellte Leitungen gibt, haben wir uns für eine getrennte Gruppenbefragung der jeweiligen Teams und Einzelbefragungen der Einrichtungsleitungen entschieden. Die Befragungen fanden im Frühjahr 2007 mit thematisch abgestimmten Leitfäden statt. (3)

Die befragten Erzieherinnen bewerten den Orientierungsplan mehrheitlich posi- tiv. Sie sehen die Chance einer Stärkung der öffentlichen Anerkennung ihrer Arbeit und einer Verdeutlichung von beruflichem Selbstverständnis. Und sie betrachten den Orientierungsplan als hilfreich für die eigene Arbeit, etwa als Leitfaden, als Strukturierungs- und Orientierungshilfe oder als Denkanstoß für konkrete Projektplanungen.

Kritische Stimmen dem Orientierungsplan gegenüber beziehen sich auf die Inhalte, die vielen Erzieherinnen als "Altbekanntes « erscheinen oder es werden Zweifel geäußert an den realen Möglichkeiten, den Plan umzusetzen, insbesondere vor dem Hintergrund schwieriger Rahmenbedingungen etwa bezogen auf Zeitverfügung und Personalausstattung. Auch konkrete Ängste offenbaren sich, wenn Erzieherinnen fürchten, durch die zeitaufwendigeren Prozesse einer individualisierten Arbeit überlastet zu werden. Zunehmende Anforderungen von Seiten der Eltern und der Schulen unterstreichen dies. Zudem fürchten sie, Orientierungspläne könnten den Einrichtungsträgern als Kontrollinstrument und als Instrument der (fachlichen) Einengung dienen.

Die inhaltliche Auseinandersetzung der Erzieherinnen mit dem Orientierungsplan, so zeigt sich in unserer Untersuchung, ist unterschiedlich weit fortgeschritten. Grundsätzlich gilt hierbei: Je intensiver die Auseinandersetzung im Team oder in einrichtungsübergreifenden Arbeitskreisen stattfindet, desto differenzierter fällt die Bewertung des Orientierungsplans aus und umso eher findet er $\mathrm{Zu}$ stimmung.

\section{Pädagogik und Bildungspläne - ein Spannungsverhältnis mit Widersprüchen}

Die meisten Bildungspläne knüpfen an die wesentlichen handlungsleitenden elementarpädagogischen Ansätze (z. B. Montessori, Reggio Pädagogik und Situ- 
ationsansatz) eher implizit als explizit an und zwar tun sie dies in Bezug auf den eigenständigen Bildungsauftrag von Kindertageseinrichtungen und hinsichtlich des Verständnisses frühkindlicher Bildungsprozesse, welches Bildung als Prozess der »Selbstbildung « fasst und die Aktivität und Selbsttätigkeit des Kindes in den Mittelpunkt stellt (vgl. Liegle 2007: 6 f.). Dennoch kommen die Bildungspläne auch im Gewand curricularer Pläne daher, wenn sie umfassend Ziele und Inhalte von Bildung beschreiben. Dies impliziert zwangsläufig die Vorstellung von zu erwerbenden Kompetenzen, gleichsam als abzuarbeitenden Katalog für die Erzieherinnen und als kindübergreifend gültig.

Diese Ambivalenz von einerseits Offenheit und andererseits relativ geschlossenem Rahmen müssen Erzieherinnen nunmehr in ihrer Arbeit allein bewältigen, indem sie eine Brücke schlagen zwischen den Interessen und Themen der Kinder und den tendenziell als Lernvorgaben aufgebauten und mit anleitenden Instruktionen versehenen Bildungsplänen. Insbesondere der baden württembergische Bildungsplan enthält wenig Anknüpfungspunkte für einen produktiven Umgang mit diesem Widerspruch, der letztlich nicht auflösbar ist. Mit Leu und Remsperger (2004) teilen wir die Auffassung, dass das Beobachten der kindlichen Bildungs- und Lernprozesse einen wesentlichen Beitrag zur produktiven Bewältigung und Gestaltung des Spannungsverhältnisses zwischen individuellen Fragen und Interessen der Kinder, den pädagogischen Ansätzen und Bildungsplänen leisten kann (vgl. Kasten »Beobachten als pädagogische Herausforderung «).

\section{Bildungs- und Entwicklungsfelder}

Die Bildungs- und Entwicklungsfelder sind in den Bildungsplänen unterschiedlich gefasst. Im baden württembergischen Orientierungsplan werden anders als in den meisten anderen Bildungsplänen die folgenden Bereiche genannt: Körper; Sinne; Sprache; Denken; Gefühl und Mitgefühl; Sinn, Werte und Religion.

Als schwierig wird in einigen Einrichtungen insbesondere das Bildungs- und Entwicklungsfeld Sinn, Werte und Religion angesehen: »Religion ist bei uns ein heikles Thema, weil ja $75 \%$ Muslime sind. « Ähnliche Situationen sind vermutlich in anderen Regionen mit hohem Migrationsanteil für Erzieherinnen vorhanden. Das heißt, es müssen Konzepte entwickelt werden, mit denen Erzieherinnen in der Lage sind, sowohl die Aussparung tern, machen den Erzieherinnen gerade in gut situierten Wohngebieten mitunter zu schaffen. Die Erzieherinnen wünschen sich deshalb, dass Land und Träger überregionale Fortbildungen für Eltern zum Thema »Orientierungsplan« anbieten.

\section{»Das Verhältnis zwischen verstehender sowie ressourcen-und defizitorientierter Beobachtung muss immer wieder neu ausgelotet werden"}

dieses Bereichs zu überwinden, als auch statisches Denken in der eigenen und fremden Kultur und Religion zu Gunsten interkultureller und interreligiöser Erziehung aufzugeben (vgl. Gogolin/Krüger Potratz 2006).

\section{Zusammenarbeit mit Eltern}

Das Thema Zusammenarbeit mit Eltern beschäftigt Erzieherinnen und die Fachöffentlichkeit schon seit Jahren, wie zahlreiche Veröffentlichungen deutlich machen. In allen Bildungsplänen wird dieser Bereich daher auch als besonders wichtig benannt. Im baden württembergischen Orientierungsplan wird sogar der Aufbau einer Erziehungspartnerschaft mit Eltern gefordert.

Bei unserer Befragung zeigte sich, dass die bisher vorhandene Zusammenarbeit mit den Eltern in den Einrichtungen unterschiedlich empfunden wird, wobei dem Charakter des Einzugsgebiets eine hohe Bedeutung für die jeweilige Einschätzung zukommt. Die Einlösung von Erziehungspartnerschaft, wird jedoch übereinstimmend als schwierig eingeschätzt, dafür werden vor allem zwei Gründe genannt:

- falsche Erwartungen der Eltern an den Kindergarten

- Probleme mit der deutschen Sprache bei Eltern von Migrantenkindern

Durch den Orientierungsplan ist die Anspruchshaltung von Eltern an den Kindergarten nach Aussage der Erzieherinnen teilweise gestiegen. Insbesondere »falsche Erwartungen « an den Kindergarten durch sehr bildungsorientierte El-
Die Erzieherinnen erhoffen sich davon eine Erleichterung der fachlichen Vertretung ihrer Arbeit gegenüber den Eltern, aber auch, dass sie nicht individuell Auseinandersetzungen über die pädagogische Haltung und Arbeit austragen müssen, die mit den Forderungen des Orientierungsplanes zusammenhängen.

Die Zusammenarbeit mit sozial benachteiligten- und Migranteneltern dagegen ist nicht erst seit der Einführung des Orientierungsplans ein Dauerproblem in deutschen Kindergärten. Die Ursache dafür liegt zumindest zum Teil in der konzeptionellen Ausrichtung des deutschenKindergartenwesens: Der Schwerpunkt besteht in der Arbeit mit Kindern, nachgeordnet gehört dann neben vielem anderen auch die Zusammenarbeit mit den Eltern dazu. Angesichts der Ergebnisse der Pisa Studie und der Forderungen im Orientierungsplan Baden Württemberg zur Erziehungspartnerschaft zwischen Eltern und Kindergarten ist es dringend erforderlich, hier neue Wege zu beschreiten, um zur Chancengerechtigkeit für Kinder beizutragen. Denkbar sind hier Entwicklungen hin zur Zusammenarbeit mit Eltern - ähnlich derjenigen in den »children centres « in Großbritannien - oder die Integration von anderen Elternbildungskonzepten für diese Gruppen, über die wir inzwischen verfügen. Bisher konnten sie in die Arbeit von Kindertageseinrichtungen u. a. schon wegen der unzureichenden Rahmenbedingungen nicht integriert werden.

Die Fachkräfte brauchen darüber hinaus insgesamt Unterstützung für eine einzugsgebietsspezifische Zusammenarbeit mit Eltern, die sehr unterschiedliche An- 
forderungen stellt. Dazu sind verbesserte strukturelle Bedingungen und neue konzeptionelle Orientierungen erforderlich.

\section{Kooperation Kindergarten und Schule}

Auch die Kooperation zwischen Kindergarten und Schule ist eine seit langem bekannte Aufgabe und folgerichtig eine zentrale Forderung des Orientierungsplans und anderer Bildungspläne. Kontakte zu Schulen bestehen in allen interviewten Einrichtungen, allerdings in unterschiedlicher Qualität. Es werden vier Problembereiche beschrieben, die alle auf der konzeptionellen, der institutionellen und der persönlichen Ebene zu bearbeiten wären:

- die Haltung der Lehrkräfte

- die mangelnde Zeit der Lehrer für Kooperationen

- die Scheinbarkeit der Kooperationslehrer

- das Bildungsverständnis beider Institutionen.
Grundsätzlich wird bemängelt, dass die Lehrkräfte als fremde Personen mit wenig zeitlicher Freistellung für diese Aufgabe in die Kindertageseinrichtung kommen und viel Druck entfalten. Sie haken ab, »es kann sprechen, es kann wahrnehmen, es kann malen ", oder sie sagen, was die Kinder alles können müssen und fordern, " puscht die mal. Da noch und dort noch ein Input. Das finde ich ganz übel, ( .) soll das ein positives Bild von sich gehe in die Schule und freue mich darauf vermitteln? «

Außerdem stellten die Erzieherinnen fest, dass in den Köpfen von so mancher Schulleitung Meinungen über Kindergärten vorhanden sind, »die überhaupt nicht mehr in unsere Zeit passen, da denkt man manchmal, wo waren diese Leute die letzten 20 Jahre «.

Interpretiert man die Daten unter dem Gesichtspunkt von Lösungsmöglichkeiten, so wird von den Erzieherinnen vorgeschlagen:

- »Die Lehrer sollten erstmal den Orientierungsplan lesen, bevor sie in die Einrichtung kommen. «
- Die Lehrkräfte sollten für die Kooperation mit den Kindertageseinrichtungen mehr Zeit zur Verfügung haben, sodass beim Übergang zwischen Kindergarten und Schule ein wirklicher Kontakt zwischen der Lehrerin oder dem Lehrer und den Kindern aufgebaut werden kann, zu der oder zu dem sie später in die Klasse kommen.

- Es sollte ein zusammenpassendes Bildungsverständnis über die Einrichtungsgrenzen hinweg für Kinder von 0 bis 14 oder 16 Jahren entwickelt werden, wie es in einigen Bildungsplänen in anderen Bundesländern gelungen ist.

- Aber vor allem muss sich die Schule ändern und zwar in der Richtung, wie sie im Orientierungsplan aufscheint, denn die Ausführungen des Orientierungsplans über Lernen in der Grundschule werden von den Erzieherinnen in der Realität von Grundschulen kaum gefunden: Eine Aussage dazu: »Der Kindergarten soll sich an der Schule orientieren, aber Schule sollte sich an einer kindorientierten Pädagogik orientieren. «

\section{Beobachten als pädagogische Herausforderung}

Die in unserer Studie befragten Erzieherinnen betonen die Gestaltung der Arbeit vom Kind und seinen Stärken und Schwächen, seinen Bedürfnissen und Interessen aus. "Beobachtungen machen die individuelle Ausgangslage des Kindes zum Ausgangspunkt pädagogischen Handelns ", so die Botschaft des baden württembergischen Orientierungsplans (MKJS Ba Wü 2006: 50).

Wahrnehmen, Beobachten und Dokumentieren von Lernund Bildungsfortschritten sowie die daran anknüpfende Planung und Gestaltung von Aktivitäten, die individuelle Bildungsprozessen anregen und unterstützen, erweisen sich als zentrale Herausforderung professionellen Handelns (vgl. MKJS Ba Wü 2006: 47).

Die Interviews vermitteln ein breit gefächertes Bild des Beobachten und Dokumentierens bei den Erzieherinnen. In der Auswertung des empirischen Materials konnten wir diesbezüglich vier typologische Muster herausarbeiten:

- Beobachten und Dokumentieren als Tätigkeit ohne direkten Bezug zur Arbeit mit den Kindern

- Beobachten und Dokumentieren mit dem Ziel, Schwächen einzelner Kinder zu identifizieren

- Beobachten und Dokumentieren als Grundlage der Darstellung der Arbeit (v. a. den Eltern gegenüber)

- Beobachten und Dokumentieren mit dem Ziel, das einzelne Kind anknüpfend an seine Stärken und unter Beachtung seiner Schwächen zu unterstützen

Im Sinne der Orientierung an den individuell besonderen Interessen und Kompetenzen von Kindern, die Leu und Remsperger (2004) als wesentliche Gemeinsamkeit der von ihnen dargestellten Beobachtungsverfahren hervorheben, kann die letztgenannte Interpretation des Beobachtens und Dokumentierens als die zentrale Bedeutungsdimension charakterisiert werden.

Insgesamt beschäftigt die Aufgabe des Beobachtens und Dokumentierens die von uns befragten Erzieherinnen stark und verunsichert sie zugleich, was nicht zuletzt Ausdruck des angesprochenen Spannungsverhältnisses von pädagogischen Ansätzen und Bildungsplänen ist aber auch von Widersprüchen in den Bildungsplänen. In den maßgeblichen Fortbildungsveranstaltungen zu den Bildungs- und Erziehungsplänen muss dies daher besonders berücksichtigt werden (vgl. Miedaner/Reinl 2007).

Bevor es dabei um methodisch technische Fragen gehen kann, die für die alltägliche Arbeit bedeutungsvoll sind, steht freilich die umfassendere Auseinandersetzung mit verschiedenen Beobachtungskonzepten (u. a. Leuvener Engagiertheitsskala, Lerngeschichten, Grenzsteine, SISMIK) und den damit verbundenen Fragen an, was, womit, wie und mit welchem Ziel beobachtet werden kann. Beobachten heißt zunächst nichts anderes als die Kinder kennenlernen und sie hierfür im Alltag wahrnehmen, sie mit Aufmerksamkeit »entdeckend beobachten «, wie Schäfer (2005: 164 ff.) ausführt.

Beobachten verbindet sich nicht per se mit der Diagnose von Störungen und Defiziten und ist nicht gleich auf Leistungsbeurteilung oder Selektion gerichtet. Dies verweist auf die Notwendigkeit, das Verhältnis zwischen verstehender sowie ressourcen- und defizitorientierter Beobachtung immer wieder neu auszuloten (vgl. Münchmeier 2004: 34 f.; Seifert 2005: $13)$.

Lore Miedaner und Heidi Reinl 
Nach Ergebnissen aus Studien über den Übergang vom Kindergarten in die Grundschule ist $\mathrm{zu}$ schließen, dass es nicht so sehr darauf ankommt, dass beide Institutionen gleich oder ähnlich sind, sondern dass ein positives- Selbstkonzept der Kinder vorhanden ist und in der Schule erhalten bleibt (vgl. Miedaner 2006).

\section{Wünsche nach Supervision}

Auffallend ist, dass sich viele Fachkräfte immer wieder eine fachliche Teambegleitung bei der Einführung des Orientierungsplans durch Mentoring oder Supervision wünschen. Dieser Wunsch ist verständlich, geht es doch um eine Veränderung der pädagogischen Haltung, um das umlernen und neu lernen und das ständige Suchen nach dem besten Weg und nicht nur um Wissen. Im Plädoyer für eine forschende Haltung der Erzieherinnen (nicht nur der Kinder) und für eine gewisse Experimentierfreude im Umgang mit dem Bildungsplan, wie sich in unseren Interviews immer wieder offenbart, sehen wir dieses Umlernen und Neulernen widergespiegelt. Wenn also eine solche Begleitung für Teams, die dies wünschen, ermöglicht werden könnte, wäre dies ohne Zweifel sinnvoll, weil es die Fachkräfte in dieser - für die Umsetzung der Bildungspläne unerlässlichen - forschenden und experimentierenden Haltung unterstützt. Dieses prozessorientierte Lernen kann durch Fortbildung kaum angegangen werden, wenngleich Fortbildung wichtige Impulse liefert.

In den meisten Regionen stößt die Beschäftigung von Supervisorinnen und Supervisoren jedoch bisher an Grenzen, weil dies bei den Finanzierungsregelungen nicht vorgesehen ist. Vergleicht man diese Situation mit der Sozialen Arbeit, so fällt auf, dass Supervision hier weitgehend zum Regelangebot für eine professionelle Arbeit gehört. Diese Form der berufsbegleitenden Qualifizierung wird den schlechter qualifizierten und schlechter bezahlten Erzieherinnen vorenthalten, obwohl sie hier um umso nötiger wären. Supervision sollte mindestens dort ergänzend eingeführt werden, wo Fachberatung zugleich Fach- und Dienstaufsicht innehat und wo die rein beratende Fachberaterin ohne Aufsichtsfunktion für zu viele Einrichtungen zuständig ist.
Zudem wäre zu überlegen, welche anderen Formen der begleitenden Unterstützung für welche Praxisfragen sinnvoll wären. Eventuell könnte ein Teil der Begleitungswünsche durch die Organisation von Tandems zwischen den Einrichtungen befriedigt werden, beispielsweise wenn es darum geht, praktische Beispiele von anderen Einrichtungen kennen zu lernen. Allerdings müsste dies durch eine Verbesserung der Rahmenbedingungen gestützt werden.

\section{Fazit}

Betrachtet man unsere Untersuchungsergebnisse unter dem Gesichtspunkt der wirklichen Verbesserung des Systems mit dem Ziel der Chancengerechtigkeit für alle Kinder, so sind grundlegende Veränderungen in Kindertageseinrichtungen notwendig.

Die Implementierung des Orientierungsplans in Baden Württemberg - wie vermutlich in anderen Bundesländern kann nicht von den Erzieherinnen allein erwartet werden. Das könnte zu rein kosmetischen Änderungen führen und nicht zu den grundlegenden Verbesserung der Arbeit in Kindertageseinrichtungen, wie Sie auch nach international vergleichenden Studien für Baden Württemberg und Deutschland erforderlich wäre.

Die Veränderung muss auf verschiedenen Ebenen erfolgen: Das Ausbildungsniveau und die berufsbegleitende Beratung der Fachkräfte in Kindertageseinrichtungen müssen ebenso verbessert werden, wie die Rahmenbedingungen und die Konzepte der institutionellen Zusammenarbeit zwischen Kindergarten und Schule. Auch muss die Schule ihre Orientierung von der Aussonderung zur Inklusion verändern. Nur so werden wir die bestmögliche Förderung für einzelne Kinder, aber auch die bestmöglichen Entwicklungschancen für unsere Gesellschaft erreichen können.

\section{Anmerkungen}

(1) Zum Beispiel für Einrichtungen für Kinder von 3 bis 6 Jahren, von 0 bis 6 Jahren, 0 bis 14 Jahren.

(2) Da an der Erhebung ausschließlich Frauen beteiligt waren, sprechen wir in diesem Beitrag von »Erzieherinnen« - und beanspruchen dennoch ausdrücklich Gültigkeit auch für die in diesem Beruf tätigen Männer.

(3) Ausführlicher zur Untersuchung: vgl. Miedaner/Reinl 2007.

\section{Literatur}

Gogolin, Ingrid/Krüger Potratz, Marianne 2006: Einführung in die Interkulturelle Pädagogik, Opladen/Farmington Hills.

Leu, Hans Rudolf/Remsperger, Regina 2004: Bildungsarbeit in der Praxis. Beobachtungsverfahren als Ergänzung zu curricularen Vorgaben, in: Wehrmann, Ilse (Hg.): Kindergärten und ihre $\mathrm{Zu}$ kunft, Weinheim/Basel/Berlin, S. 167180.

Liegle, Ludwig 2007: Pädagogische Konzepte und Bildungspläne - wie stehen sie zueinander?, in: Kindergarten heute, H. 1, S. 6-12.

Miedaner, Lore 2006: Anschlussfähige Bildung zwischen Elementar- und Primarbereich Teil II, in: Kita aktuell, 15. Jg., Nr.12, S. 244-248.

Miedaner, Lore/Reinl, Heidi 2007: Erwartungen an die Umsetzung des Orientierungsplanes. Der Blickwinkel der Erzieherinnen, in: Kita Aktuell BW, H. 9.

MKJS - Ministerium für Kultus, Jugend und Sport Baden Württemberg 2006: Orientierungsplan für Bildung und Erziehung für die baden württembergischen Kindergärten, Pilotphase, Weinheim/Basel.

Münchmeier, Richard 2004: Bildung ist mehr als Lernen - Konzepte für ein angemessenes Bildungsverständnis im Vorschulbereich, in: Friedrich Ebert Stiftung Landesbüro Sachsen Anhalt 2004: Die Chancen der frühen Jahre nutzen - Lernen und Bildung im Vorschulalter, Magdeburg, S. 24-41.

Schäfer, Gerd E. 2005: Aufgaben frühkindlicher Bildung, in: ders. (Hg.): Bildung beginnt mit der Geburt. Ein offener Bildungsplan für Kindertageseinrichtungen in Nordrhein Westfalen, Weinheim/Basel, S. 75-178.

Seifert, Brigitte 2005: Die Gesundheit der Kinder lässt zu wünschen übrig, in: DJI Bulletin, 74, S. 12 f. 